\title{
Acromicric dysplasia: long term outcome and evidence of autosomal dominant inheritance
}

Département de Génétique, Hôpital

Necker-Enfants

Malades, 149 rue de

Sèvres, 75015 Paris,

France

L Faivre

M Le Merrer

A Munnich

P Maroteaux

V Cormier-Daire

Service de Génétique, Hôpital Robert Debré, Paris, France

C Baumann

Service

d'Endocrinologie et Diabètologie, Hôpital Robert Debré, Paris,

France

M Polak

Endocrinologie Pédiatrique, Hôpital Debrousse, Lyon, France

P Chatelain

Polyclinique Pédiatrique, American Memorial Hospital,

Reims, France

V Sulmont

Clinique Médicale Infantile, Hôpital Saint Antoine, Lille, France

J Cousin

Médecine Infantile, CHRU La Tronche, Grenoble, France

M Bost

Génétique Médicale, Hôpital Edouard Herriot, Lyon, France M P Cordier

Clinical Genetics Center, The Children's Hospital of Philadelphia, PA, USA E Zackai

K Russell

Chirurgie Orthopédique Pédiatrique, Hôpital Necker-Enfants

Malades, Paris, France G Finidori

J C Pouliquen

Correspondence to: Dr Cormier-Daire, cormier@necker.fr

Revised version received 8 August 2001

Accepted for publication

22 August 2001

L Faivre, M Le Merrer, C Baumann, M Polak, P Chatelain, V Sulmont, J Cousin, M Bost, M P Cordier, E Zackai, K Russell, G Finidori, J C Pouliquen, A Munnich, P Maroteaux, V Cormier-Daire

\begin{abstract}
Acromicric dysplasia is a rare bone dysplasia characterised by short stature, short hands and feet, normal intelligence, mild facial dysmorphism, and characteristic $x$ ray abnormalities of the hands. Only a very small number of children with this condition have been reported so far. Here we report on a series of 22 patients including 10 boys and 12 girls with acromicric dysplasia. Length was normal at birth and height fell progressively off the centiles postnatally. The mean adult height was $130 \mathrm{~cm}(133 \mathrm{~cm}$ in males, 129 cm in females). The hands, feet, and limbs were short and OFC was normal. Intelligence was normal and mild dysmorphic features were noted. Other occasional features included well developed muscles, a hoarse voice, generalised joint limitation in some patients, frequent ear, tracheal, and respiratory complication, and spine abnormalities. Long term follow up showed that facial dysmorphism was less obvious in adults and that carpal tunnel syndrome was frequent in older patients. Apart from short metacarpals and phalanges, internal notch of the second metacarpal, external notch of the fifth metacarpal, and internal notch of the femoral heads, there were no major $x$ ray abnormalities. No major complications, such as cardiac disease or major orthopaedic problems, occurred in the course of the disease. The condition appeared to be sporadic in 16 cases but the observation of vertical transmission in three families was consistent with an autosomal dominant mode of inheritance. (F Med Genet 2001;38:745-749)
\end{abstract}

Keywords: acromicric dysplasia; geleophysic dysplasia; long term outcome; autosomal dominant inheritance

In 1986, Maroteaux et al described a novel bone dysplasia in six unrelated children presenting with short stature, short hands and feet, normal intelligence, mild facial dysmorphic features, and characteristic $x$ ray abnormalities of the hands. They called this condition "acromicric dysplasia". ${ }^{1}$ Only a few cases with features of acromicric dysplasia have been reported since then, but all children presented overlapping features with geleophysic dysplasia or Moore-Federman syndrome..$^{2-4}$ In the last 14 years, we have ascertained 18 additional cases of acromicric dysplasia in 15 families from five different countries and followed up four of the previously reported cases. Here we describe the clinical data and $x$ ray features of these patients, with particular emphasis on the long term outcome in 10 adult patients, and provide further support for the autosomal dominant inheritance of this condition.

\section{Patients and methods}

Twenty-two patients (12 girls, 10 boys) from 15 families and five different countries were included in our study (table 1). Their ages ranged from 4.5 to 53 years with a mean age of 19 years. The diagnosis of acromicric dysplasia was based on the observation of short stature, short hands and feet, short and stubby metacarpals and phalanges with an external notch on the second metacarpal and an internal notch on the fifth metacarpal, and mild deformity of the femoral heads. Sixteen of our 22 patients were seen at least once in the medical genetics clinic of Necker-Enfants Malades Hospital. Questionnaires were sent to the referring physicians and patients were contacted directly for follow up information. Eight patients were identified through medical reports sent to our clinic for advice and questionnaires were sent to the referring physician for additional information.

\section{Results}

The mean length at birth was $48.6 \mathrm{~cm}(48.8$ $\mathrm{cm}$ in boys, $48.5 \mathrm{~cm}$ in girls) and the mean adult height was $130 \mathrm{~cm}(133 \mathrm{~cm}$ in males, 129 $\mathrm{cm}$ in females), with a range from 115 to 140 $\mathrm{cm}$. In all cases, the height fell progressively off the centiles (fig 1). The limbs were short and OFC was normal. One case showed significant improvement after a limb lengthening procedure and one patient received growth hormone with no significant effect on final height. Short stature was generally well accepted by the patients and their quality of life was not affected. Adults did well in their professional lives and intelligence was normal.

Facial dysmorphism was very similar in all cases (fig 2). It included a round face (17/22 cases), narrow palpebral fissures (17/22 cases), well defined eyebrows (14/22 cases), long eyelashes (14/22 cases), a bulbous nose with anteverted nostrils (19/22 cases), a long and prominent philtrum (16/22 cases), and thick lips (13/22 cases) with a small mouth $(12 / 22$ cases). Facial dysmorphism seemed less obvious in older patients.

Occasional features included joint limitation (13/20 cases), kyphoscoliosis or lordosis (12/20 cases), recurrent ear, tracheal, and respiratory complications (otitis, tracheal infections, and 
Table 1 Clinical features in acromicric dysplasia patients

\begin{tabular}{|c|c|c|c|c|c|c|c|c|c|c|c|c|c|c|c|c|c|c|c|c|c|c|c|}
\hline & \multicolumn{6}{|c|}{ Familial cases } & \multicolumn{16}{|c|}{ Sporadic cases } & \multirow[b]{2}{*}{ Total } \\
\hline & 1 & 2 & 3 & 4 & 5 & 6 & 7 & 8 & 9 & 10 & 11 & 12 & 13 & 14 & 15 & 16 & 17 & 18 & 19 & 20 & 21 & 22 & \\
\hline Ethnic origin & Fr & $\mathrm{Fr}$ & Fr & $\mathrm{Fr}$ & A & A & $\mathrm{Fr}$ & Fr & $\mathrm{Fr}$ & $\mathrm{Fr}$ & $\mathrm{Fr}$ & A & $\mathrm{Fr}$ & USA & USA & $\mathrm{Sp}$ & $\mathrm{Sp}$ & Fr & It & $\mathrm{Fr}$ & $\mathrm{Fr}$ & It & \\
\hline Sex & $\mathrm{F}$ & M & $\mathrm{F}$ & $M$ & $\mathrm{~F}$ & $M$ & $\mathrm{~F}$ & $\mathrm{M}$ & $\mathrm{F}$ & $\mathrm{F}$ & $M$ & $\mathrm{~F}$ & $\mathrm{~F}$ & $\mathrm{~F}$ & M & $\mathrm{F}$ & $\mathrm{F}$ & $\mathrm{M}$ & M & $\mathrm{F}$ & M & M & $10 \mathrm{M} / 12 \mathrm{~F}$ \\
\hline Familial case & + & + & + & + & + & + & - & - & - & - & - & - & - & - & - & - & - & - & - & - & - & - & $6 / 22$ \\
\hline Length at birth $(\mathrm{cm})$ & 49 & 47 & 49 & 48 & 48 & 46 & 48 & 49 & 47.5 & 49 & 48 & 49 & 46 & 48 & 49.5 & NA & NA & 49 & 51 & 51 & 50 & 50 & 48.6 \\
\hline \multicolumn{24}{|l|}{ Follow up } \\
\hline Age (years) & 48 & 21 & 53 & 22 & 33 & 4 & 8 & 12 & 19 & 20 & 14 & 11 & 9 & 16 & 9.5 & 7 & 8 & 15 & 11 & 21 & 33 & 18 & 18.8 \\
\hline Height $(\mathrm{cm})$ & 132 & 138 & 120 & 128 & 140 & 93 & 98 & 112 & 115 & 135 & 120 & 118 & 116 & 138 & 111 & 97.5 & 92 & 114 & 106 & $\begin{array}{l}120 \\
+20^{\star}\end{array}$ & 140 & 125 & $\begin{array}{l}130 \text { in } \\
\text { adults }\end{array}$ \\
\hline Abnormal voice & - & - & + & + & + & - & - & - & + & - & - & NA & NA & - & NA & NA & NA & NA & NA & + & - & - & $5 / 15$ \\
\hline Well developed muscles & - & + & + & + & - & - & + & - & - & + & - & - & NA & - & NA & NA & + & + & NA & + & + & - & $10 / 18$ \\
\hline Joint limitation & - & $\mathrm{H}, \mathrm{Fi}$ & All & All & - & - & $\mathrm{E}, \mathrm{Fi}$ & - & $S$ & $\mathrm{Fi}$ & - & $\mathrm{Fi}$ & - & - & $\mathrm{E}, \mathrm{Fi}$ & NA & NA & $\mathrm{H}, \mathrm{E}$ & $\begin{array}{l}\text { E,F, } \\
\text { H,K }\end{array}$ & $\begin{array}{l}\mathrm{E}, \mathrm{Fi} \text {, } \\
\mathrm{H}\end{array}$ & $\mathrm{H}$ & $\begin{array}{l}\text { E,H, } \\
\mathrm{Fi}\end{array}$ & $13 / 20$ \\
\hline Facial dysmorphism & + & + & + & + & + & + & + & + & + & + & + & + & + & + & + & + & + & + & + & + & + & + & $22 / 22$ \\
\hline Heart malformations & - & - & - & - & - & - & - & - & + & - & + & - & - & - & + & - & - & + & - & - & - & - & $4 / 22$ \\
\hline Hepatomegaly & - & - & - & - & - & - & - & - & - & - & - & - & - & - & - & - & - & - & - & - & - & - & $0 / 22$ \\
\hline Eye abnormalities & + & + & + & - & + & - & - & - & + & - & + & - & - & + & - & NA & NA & - & - & + & - & - & $8 / 20$ \\
\hline Ear abnormalities & + & + & + & - & - & - & + & - & - & - & - & - & - & + & + & NA & NA & - & - & - & - & - & $6 / 20$ \\
\hline Tracheal, respiratory & - & + & + & + & - & - & - & - & + & - & - & - & - & + & - & - & - & + & - & + & - & - & $7 / 22$ \\
\hline Spine abnormalities & + & - & + & + & + & - & - & + & + & + & + & - & + & - & + & NA & NA & + & - & + & - & - & $12 / 20$ \\
\hline Carpal tunnel & + & - & + & - & + & - & - & - & - & - & - & - & - & - & - & - & - & - & - & - & - & - & $3 / 22$ \\
\hline
\end{tabular}

Fr, France; A, Africa; Sp, Spain; It, Italy.

$\mathrm{F}$, female; $\mathrm{M}$, male.

H, hips; Fi, fingers; E, elbows; K, knees; S, shoulders.

NA, not available.

$\star+20 \mathrm{~cm}$ after limb lengthening procedure.

asthma, 10/20 cases), well developed muscles (10/18 cases, fig 3$)$, eye refraction defects (hypermetropia, myopia, and astigmatism, $8 / 20$ cases), abnormally hoarse voice $(5 / 15$ cases), heart malformations (atrial septal defect and bicuspid aorta, 4/22 cases), and carpal tunnel syndrome in older patients (3/22 cases, table 1). No pain, thickened skin, or hepatomegaly was observed. Finally, irregular leg length (1/22 cases), osteoarthritis (1/22 cases), clenched fingers (1/22 cases), Brown syndrome (limitation of upward gaze) (1/22 cases), pulmonary hypertension ( $1 / 22$ cases), autoimmune diabetes ( $1 / 22$ cases), hepatitis ( $1 / 22$ cases), delayed puberty ( $1 / 22$ cases), and precocious puberty ( $1 / 22$ cases) were occasionally noted.

Besides short metacarpals and phalanges, internal notch of the second metacarpal, and external notch of the fifth metacarpal, other consistent features were shortened fourth and fifth metacarpals and markedly delayed bone age. Mild pointing of the second to fifth metacarpals was observed in $15 / 22$ cases and cone shaped epiphyses in $5 / 22$ cases. $X$ rays of the older patients showed that the notches of the hands disappeared in adulthood (fig 4). An internal notch of the femoral heads was consistently observed and persisted in adulthood (fig 5). There was neither osteopenia nor
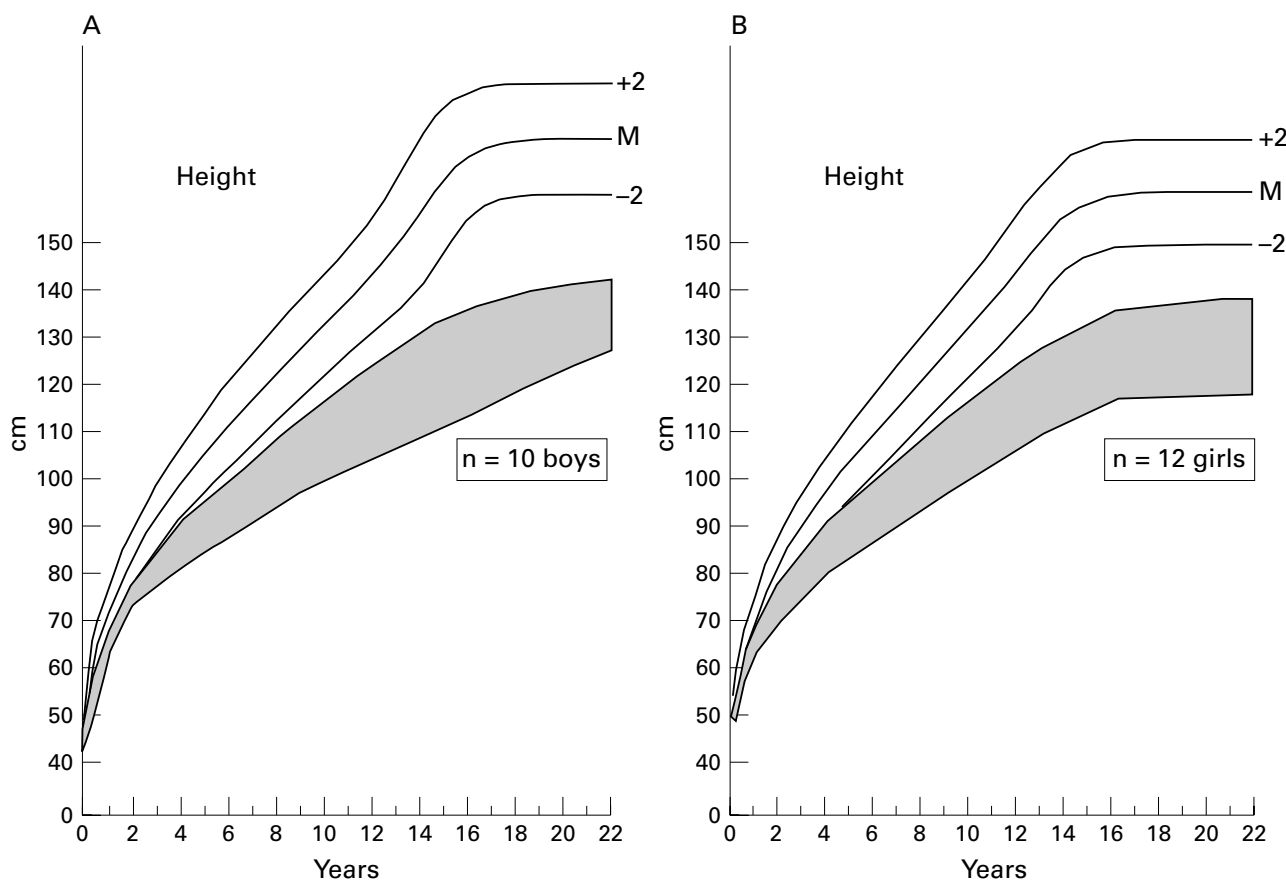

Figure 1 Maximum and minimum growth curve in 22 acromicric dysplasia patients. Birth length ranged from 45 to 51 $\mathrm{cm}$. Adult height ranged from 115 to $140 \mathrm{~cm}$. 

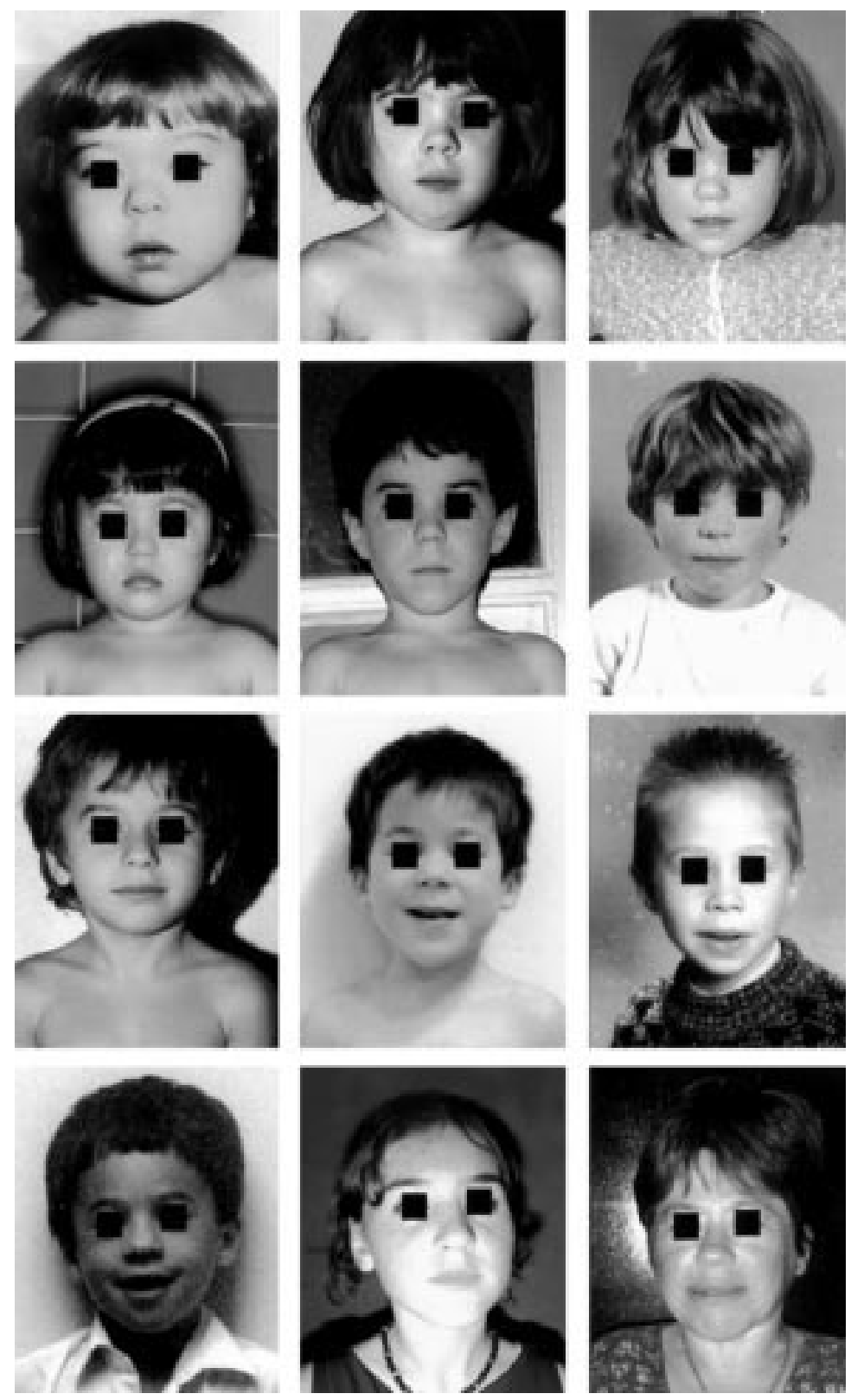

Figure 2 Facial dysmorphism in 12 patients with acromicric dysplasia. Note the round face, narrow palpebral fissures, well defined eyebrows and long eyelashes, a bulbous nose with anteverted nostrils, a long and prominent philtrum, and thick lips with a small mouth. The facial dysmorphism seems less obvious in adults.
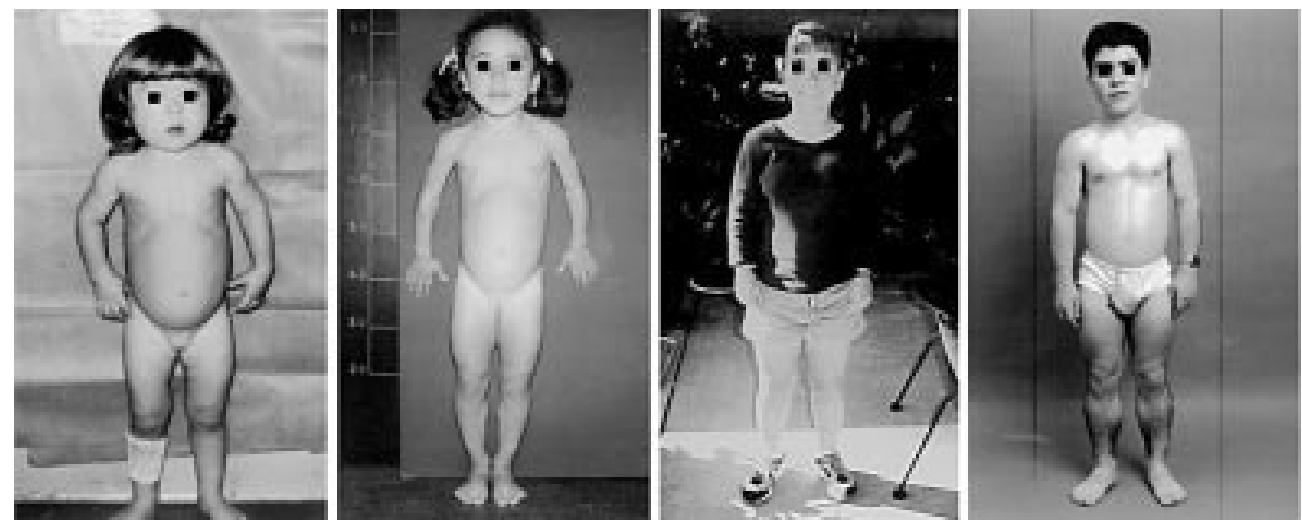

\section{Discussion}

Here we report progressive dwarfism, short hands and feet, normal intelligence, and mild dysmorphic features in 22 patients with acromicric dysplasia. Additional clinical features of the syndrome were identified in this study, namely well developed muscles, hoarse voice, generalised joint limitations, frequent ear, tracheal, and respiratory complications, and spine abnormalities. Long term follow up showed that facial dysmorphism was less obvious in adults and that carpal tunnel syndrome was frequent in older patients. The severity of dwarfism was quite variable, but consistently below $3 \mathrm{SD}$, and $\mathrm{OFC}$ was consistently normal. Life expectancy was normal and no major complications, such as cardiac disease or major orthopaedic problems, occurred in the course of the disease. Only mild $x$ ray abnormalities were noted and notches of the hands disappeared in adulthood.

Several acromicric dysplasia patients with features of geleophysic dysplasia and MooreFederman syndrome, namely stiff joints, thickened skin, and hepatomegaly, have been reported, raising the question of whether geleophysic dysplasia, Moore-Federman syndrome, and acromicric dysplasia could be the same entity. ${ }^{2-4}$ Moreover, short stature with short hands and feet, normal birth length with reduced postnatal growth rate, and delayed bone age are common features in all three conditions. Table 2 summarises the differences between the three entities. First, thickened skin

Figure 3 Front views of five acromicric dysplasia patients. The limbs, hands, and feet were short and well developed muscles were noted in several patients. 

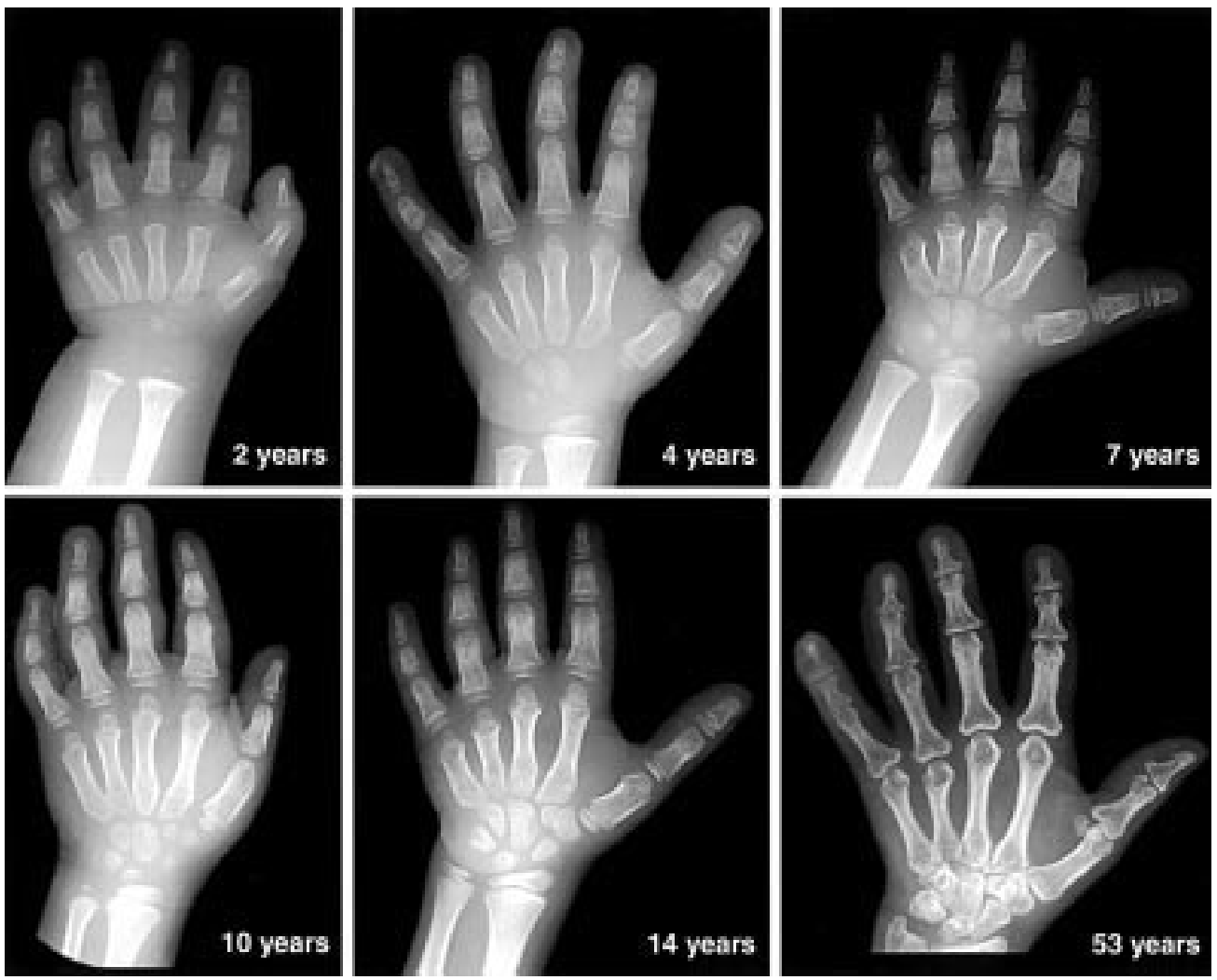

Figure 4 X rays of the hands in acromicric dysplasia. Note the short metacarpals and phalanges, the internal notch of the second metacarpal and the external notch of the fifth metacarpal, short fourth and fifth metacarpals, mild pointing of the second to fifth metacarpals, and markedly delayed bone age. Note that hand notches disappeared in adulthood.

and hepatomegaly are features of both geleophysic dysplasia and Moore-Federman syndrome, but are never observed in acromicric dysplasia. Second, cardiac valve dysplasia is observed in geleophysic dysplasia only. ${ }^{5}$ Third, while life expectancy and functional prognosis
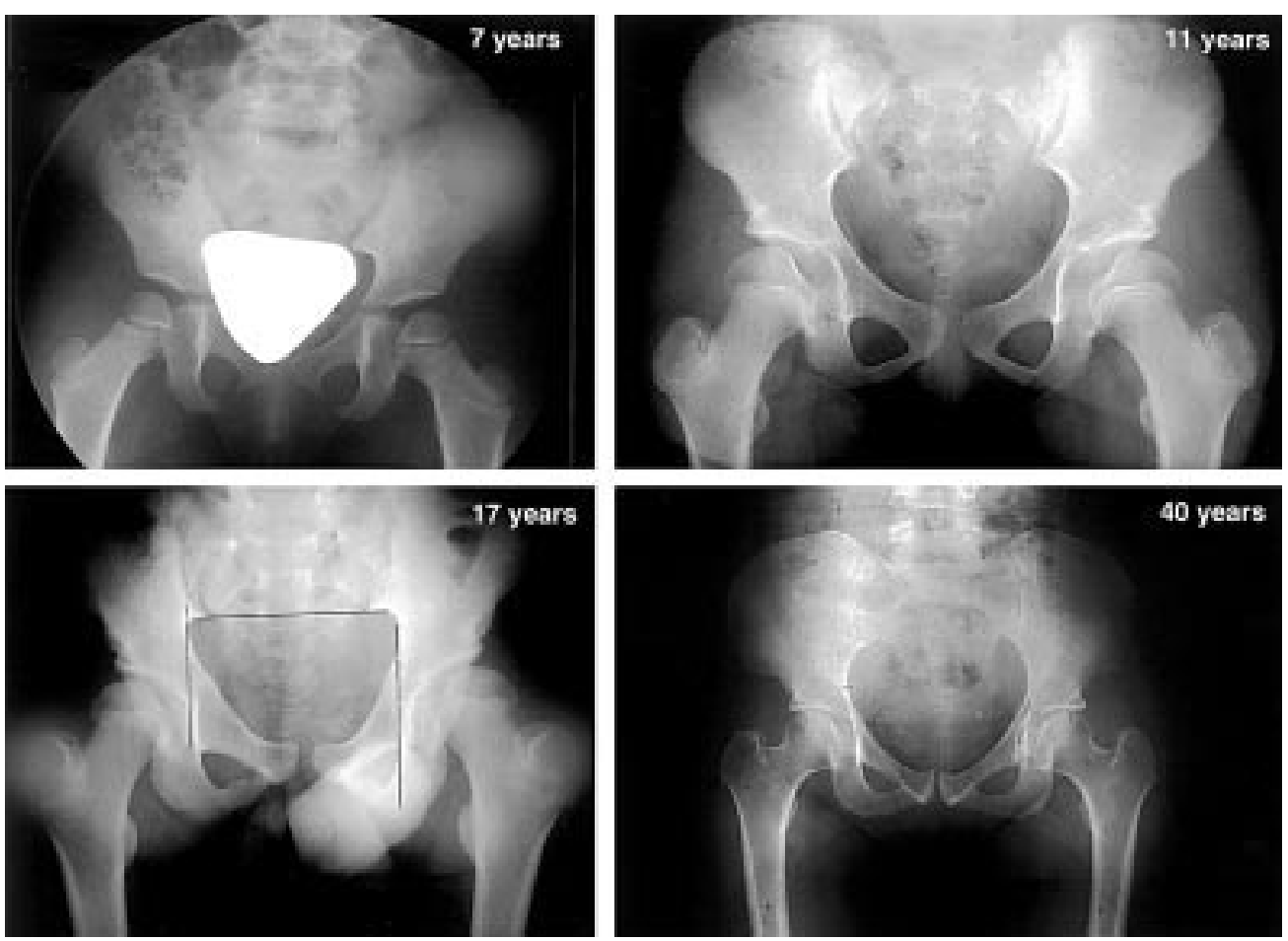

Figure $5 X$ rays of the pelvis in acromicric dysplasia. Note the internal notch of the femoral heads. This notch did not disappear with age.

are good in acromicric dysplasia and MooreFederman syndrome, they are poor in geleophysic dysplasia. ${ }^{6}$ In addition, the internal notch of the femoral heads is specific to acromicric dysplasia. Also, histological signs of storage disorder have been found in geleophysic 


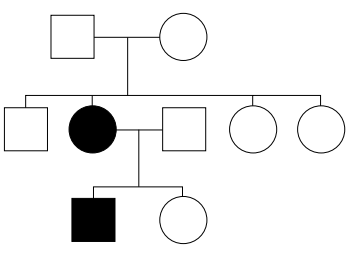

Family 1

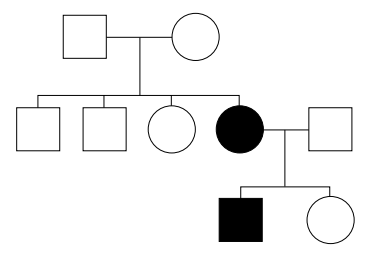

Family 2

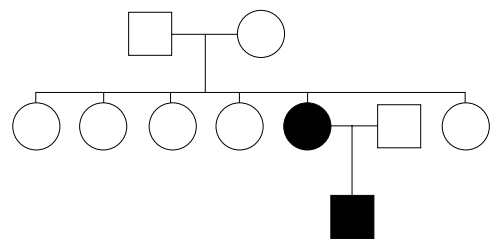

Family 3

Figure 6 Vertical transmission of the disease in the three familial cases of acromicric dysplasia.

dysplasia. ${ }^{48}$ Finally, the mode of inheritance is different in the three conditions: autosomal dominant in acromicric dysplasia and MooreFederman syndrome ${ }^{910}$ and autosomal recessive in geleophysic dysplasia. ${ }^{112}$ We therefore believe that at least geleophysic dysplasia is a distinct entity from acromicric dysplasia. The differences between acromicric dysplasia and Moore-Federman syndrome are minor and the existence of only two reports of MooreFerderman syndrome limits the knowledge of the disease. ${ }^{39}$ However, the hypothesis of clinical variability of the same entity with hepatomegaly and thickened skin being more obvious with age is not supported by the long term follow up of four patients aged 33 years or more in our series.

The pathogenesis of the disease is unknown. Cartilage histology was performed in two of the first six cases with acromicric dysplasia ${ }^{1}$ and showed some disorganisation of the growth

Table 2 Similarities and differences between acromicric dysplasia, Moore-Federman syndrome, and geleophysic dysplasia

\begin{tabular}{llll}
\hline & Acromicric dysplasia & Moore-Federman ${ }^{10}$ & Geleophysic dysplasia $^{5}$ \\
\hline Major distinctive features & & & + \\
Thickened skin & - & + & + \\
Cardiac valve disease & - & - & Poor \\
Prognosis & Good & Deformed & Dysplastic \\
Femoral heads & Notch & NR & Storage \\
Histology & Normal & AD & AR \\
Inheritance & AD & & \\
Minor distinctive features & & Moderate $-10 / 11$ & Severe $-21 / 23$ \\
Joint contractures & Mild $-13 / 20$ & $5 / 11$ & $14 / 21$ \\
Hepatomegaly & $0 / 22$ & $11 / 11$ & $16 / 21$ \\
Height <3rd centile & $22 / 22$ & NR & $4 / 14$ \\
Speech delay & $0 / 22$ & Hoarse & High pitched \\
Voice & Hoarse & $9 / 11$ & Possible \\
Eye abnormalities & $8 / 20$ & & \\
Similarities & & Often normal & Often normal \\
Birth length & Often normal & + & + \\
Reduced postnatal & + & & \\
growth speed & & NR & Frequent \\
Delayed bone age & Consistent & NR & + \\
$\begin{array}{l}\text { Susceptibility to ENT } \\
\text { and respiratory infections }\end{array}$ & + & & \\
\hline
\end{tabular}

NR: not reported.

ENT: ear, nose, and throat. zone with islands of cells, some of them degenerated, an abnormal organisation of collagen forming thick rims around the cells, and wide fibres in the inter-territorial matrix, and a large accumulation of glycogen in most chondrocytes. These results do not suggest which are the candidate genes in this disorder.

In conclusion, we believe that acromicric dysplasia is a genuine entity, distinct from geleophysic dysplasia and, here, we provide additional support for the autosomal dominant inheritance of acromicric dysplasia. It is hoped that further familial cases will help in the mapping and identifying of the disease gene.

We would like to acknowledge Damien Bonnet for his heart ultrasound expertise, Francis Brunelle for helpful discussion on $x$ rays, Sylvie Fraitag and Catherine Prost for histological studies and electron microscopy of the skin, and Y Deris for photographic assistance.

1 Maroteaux P, Stanescu R, Stanescu V, Rappaport R. Acromicric dysplasia. Am f Med Genet 1986;24:447-9.

2 Hennekam RC, van Bever Y, Oorthuys JW. Acromicric dysplasia and geleophysic dysplasia: similarities and differences. Eur F Pediatr 1996;155:311-14.

3 Winter RM, Patton MA, Challener J, Mueller RF, Baraitser $M$. Moore-Federman syndrome and acromicric dysplasia: are they the same entity? 7 Med Genet 1989;26:320-5.

4 Lipson AH, Kan AE, Kozlowski K. Geleophysic dysplasia B acromicric dysplasia with evidence of glycoprotein storage. Am 7 Med Genet Suppl 1987;3:181-9.

5 Santolaya JM, Groninga LC, Delgado A, Monasterio JL, Santolaya JM, Groninga LC, Delgado A, Monasterio JL,
Camarero C, Bilbao FJ. Patients with geleophysic dysplasia are not always geleophysic. Am f Med Genet 1997;72:8590

6 Wraith JE, Bankier A, Chow CW, Danks DM, Sardharwalla IB. Geleophysic dysplasia. Am f Med Genet 1990;35:153-6.

7 Shohat M, Gruber HE, Pagon RA, Witcoff LJ, Lachman R, Ferry D, Flaum E, Rimoin D. Geleophysic dysplasia: a storage disorder affecting the skin, bone, liver, heart, and trachea. F Pediatr 1990;117:227-32.

8 Pontz BF, Stöb H, Henschke F, Freisinger P, Karbowski A, Spranger J. Clinical and ultrasound findings in three patients with geleophysic dysplasia. Am f Med Genet 1996; 63:50-4.

9 Moore WT, Federman DD. Familial dwarfism and "stiff joints". Report of a kindred. Arch Intern Med 1965;115:

10 Fell JME, Stanhope R. Reviving the Moore-Federman syndrome. F $R$ Soc Med 1993;86:52-3.

11 Spranger J, Gilbert EF, Arya S, Hoganson GMI, Opitz JM. Geleophysic dysplasia. Am F Med Genet 1984;19:501-6.

12 Titomanlio L, Della Casa R, Lecora M, Farina V, Sebastio G, Andria G, Parenti G. Geleophysic dysplasia: 7-year follow-up study of a patient with an intermediate form. $\mathrm{Am}$ f Med Genet 1999;86:82-5. 